Original Article

\title{
Lumbar curvature: a previously undiscovered standard of attractiveness
}

\author{
David M.G. Lewis $^{\text {a,* }}$, Eric M. Russell ${ }^{\text {b, } 1}$, Laith Al-Shawaf ${ }^{\text {c,d,1 }}{ }^{\text {, David M. Buss }}{ }^{\text {d }}$ \\ a Bilkent University, Ankara, Turkey \\ b The University of Texas at Arlington, Arlington, TX, USA \\ c American University of Beirut, Beirut, Lebanon \\ d The University of Texas at Austin, Austin, TX, USA
}

\section{A R T I C L E I N F O}

Article history:

Initial receipt 27 September 2014

Final revision received 29 January 2015

\section{Keywords:}

Evolutionary psychology

Mate preferences

Physical attractiveness

Lumbar curvature

\begin{abstract}
A B S T R A C T
This paper reports independent studies supporting the proposal that human standards of attractiveness reflect the output of psychological adaptations to detect fitness-relevant traits. We tested novel a priori hypotheses based on an adaptive problem uniquely faced by ancestral hominin females: a forward-shifted center of mass during pregnancy. The hominin female spine possesses evolved morphology to deal with this adaptive challenge: wedging in the third-to-last lumbar vertebra. Among ancestral women, vertebral wedging would have minimized the net fitness threats posed by hypolordosis and hyperlordosis, thereby creating selective pressures on men to prefer such women as mates. On this basis, we hypothesized that men possess evolved mate preferences for women with this theoretically optimal angle of lumbar curvature. In Study 1, as hypothesized, men's attraction toward women increased as women's lumbar curvature approached this angle. However, vertebral wedging and buttock mass can both influence lumbar curvature. Study 2 thus employed a forced-choice paradigm in which men selected the most attractive woman among models exhibiting the same lumbar curvature, but for different morphological reasons. Men again tended to prefer women exhibiting cues to a degree of vertebral wedging closer to optimum. This included preferring women whose lumbar curvature specifically reflected vertebral wedging rather than buttock mass. These findings reveal novel, theoretically anchored, and previously undiscovered standards of attractiveness.
\end{abstract}

(c) 2015 Elsevier Inc. All rights reserved.

\section{Introduction}

A prevalent historical view in the behavioral sciences has been that human standards of beauty are arbitrary and dependent on sociocultural processes alone (e.g., Berscheid \& Walster, 1974; Langlois et al., 1987). This view contrasts with evidence from non-human species that an organism's attractiveness as a mate is linked to traits that help solve adaptive challenges related to survival and reproduction (Norris, 1993; Petrie, 1994; Birkhead \& Fletcher, 1995; Stacey, Eileen, Rebecca, \& Kevin, 2011). We report independent studies testing the overarching proposal that human standards of attractiveness reflect the output of evolved psychological mechanisms designed to detect fitness-relevant traits (Symons, 1995; Thornhill \& Gangestad, 1999; Gangestad \& Scheyd, 2005; Sugiyama, 2005; Singh \& Singh, 2011). Specifically, we tested novel hypotheses based on an adaptive challenge uniquely faced by ancestral hominin females: a bipedal fetal load.

\footnotetext{
* Corresponding author. Department of Psychology, Bilkent University, 06800 Bilkent, Ankara, Turkey. Tel.: +1206525 4479.

E-mail address: david@davidmglewis.com (D.M.G. Lewis).

${ }^{1}$ The contributions of the second and third authors were equal to one another.
}

\subsection{Pregnancy and lumbar curvature}

The combination of bipedalism and increased abdominal mass during pregnancy uniquely posed ancestral hominin females with the adaptive challenge of a forward-shifted center of mass (COM) during pregnancy. If this COM were not moved back over the hips, ancestral women would have been subjected to a nearly $800 \%$ increase in hip torque during pregnancy (Whitcome, Shapiro, \& Lieberman, 2007). The muscular fatigue and lower back pain resulting from this hip torque would have impaired foraging efficiency (Whitcome et al., 2007). This would have left women and their families-including fetus, offspring, and mate-at risk of nutritional stress (Marlowe, 2003; Whitcome et al., 2007). Selection would therefore have favored morphological adaptation in women that enabled them to shift the gravid COM back over the hips.

The female hominin spine ${ }^{1}$-but not the male hominin spinepossesses evolved morphology to deal with this adaptive challenge: women possess wedging in the third-to-last lumbar vertebra (Whitcome et al., 2007). This wedging helps pregnant women shift their COM back over their hips, thereby reducing hip torque by over 90\% (Whitcome et al., 2007). The inability to shift the COM back over

\footnotetext{
1 This includes the spine of females from the Homo genus as well as that of females from extinct bipedal hominin lineages (Whitcome et al., 2007).
} 

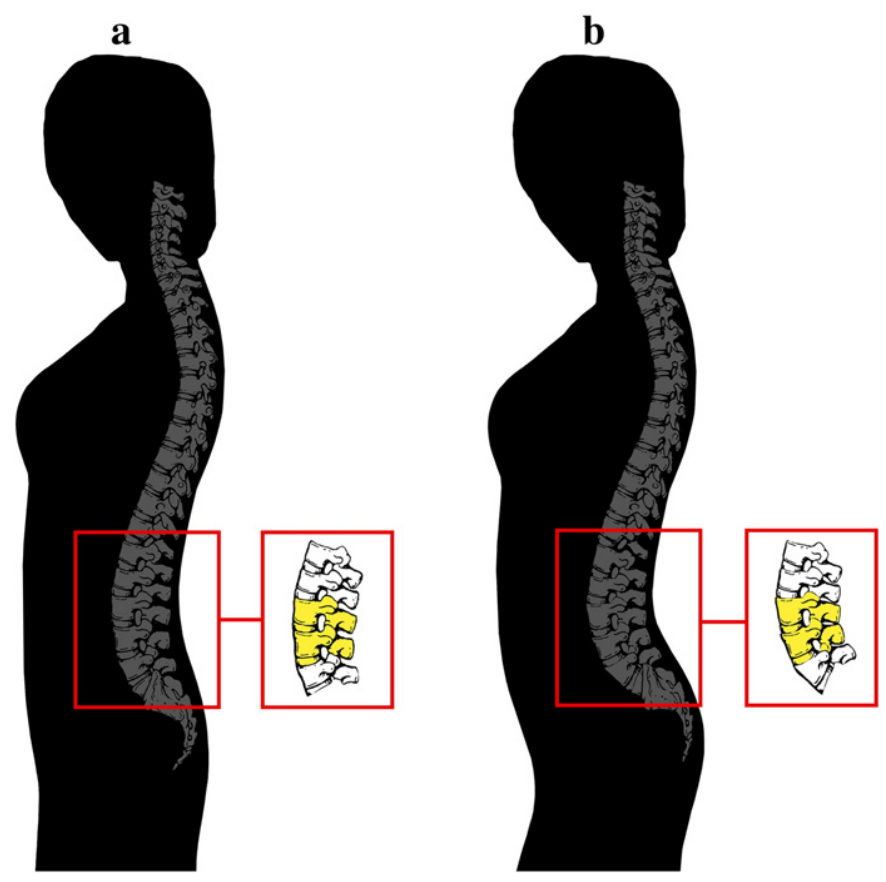

Fig. 1. A woman with (a) less vertebral wedging at the third-to-last lumbar vertebra (modal L3 depicted), and (b) greater wedging, resulting in a more acute angle of lumbar curvature.

the hip joints, on the other hand, results in sustained contraction of the lower back muscles, which increases risk of fatigue and injury (White \& Punjabi, 1990).

Indeed, insufficient lumbar curvature (hypolordosis) is associated with lower back pain (Rath \& Rath, 1997), but so is excessive lumbar curvature (hyperlordosis; Christie, Kumar, \& Warren, 1995; Magnora, 1975). These countervailing forces would have resulted in stabilizing selection on ancestral women's spines. Selection would have favored vertebrae in women that were (1) sufficiently wedged to center the gravid COM back over the hips, but nonetheless (2) provided sufficient skeletal reinforcement to prevent hyperlordosis, fatigue, and spinal injury (White \& Punjabi, 1990; Whitcome et al., 2007).

\subsection{Women's lumbar curvature and male mating psychology}

The fitness benefits experienced by ancestral women with a beneficial intermediate degree of lumbar wedging between hypolordosis and hyperlordosis would have created the background selective conditions for the evolution of a male mate preference for such women. Men who preferred and selected these women as mates would have gained several key fitness benefits, including having a mate who was less vulnerable to spinal injuries, better at foraging during pregnancy, and better able to sustain multiple pregnancies without debilitating injury. On this basis, we advance the hypothesis that selection fashioned psychological adaptations in men to detect cues to lumbar vertebral wedging in women and regulate mating attraction accordingly.

Ancestral men could not have directly observed potential mates' vertebrae, so their detection of vertebral wedging had to be based on observable cues. One cue to lumbar vertebral wedging is the curvature of the lower back (George, Hicks, Nevitt, Cauley, \& Vogt, 2003). Wedging in the lumbar vertebrae extends the tailbone relative to the rest of the spine, resulting in a more acute angle between the thoracic back and buttocks (see Fig. 1). ${ }^{2}$

\footnotetext{
${ }^{2}$ Consistent with this, the sex difference in vertebral wedging is associated with a sex difference in external lumbar curvature (Fernand \& Fox, 1985; women: mean $=47.19^{\circ}$, men: mean $=43.25^{\circ}$, Cohen's $d=.35$ ).
}

We therefore advanced the hypothesis that men possess evolved mate preferences for lumbar curvature in women. Because the theoretically optimal degree of vertebral wedging for women is one that minimizes the net fitness threats posed by hypolordosis and hyperlordosis, we hypothesized that men should possess an evolved preference for women with an angle of lumbar curvature maximally distant from these countervailing threats, which orthopedic medical literature indicates is approximately $45.5^{\circ}$ (see Fernand \& Fox, 1985).

\section{Study 1: lumbar curvature and attractiveness}

Men rated the attractiveness of female models varying in lumbar curvature. Unlike previous research, we used profile rather than frontal images; the latter cannot capture important cues available only from alternative perspectives (Marlowe, Apicella, \& Reed, 2005), such as lumbar curvature from the side. Consequently, the current studies are (1) among the few to employ non-frontal views, and (2) the first to assess the influence of lumbar curvature on physical attractiveness.

\subsection{Method}

\subsubsection{Participants}

One hundred two men $\left(M_{\text {age }}=19.00\right.$ years, $S D_{\text {age }}=2.41$, age range: 17-34 years) were recruited from the psychology subject pool at The University of Texas at Austin. Participants received course credit for participation.

\subsubsection{Photographic stimuli and attractive ratings}

Fifteen images were generated in Adobe Photoshop by manipulating the angle of lumbar curvature of female targets. For each target, we generated five morphs of varying angles of lumbar curvature (see Fig. 2). These stimuli captured the naturally occurring range of lumbar curvature in the population (stimuli range: $14-69^{\circ}$; see Fernand \& Fox, 1985). The targets' lumbar curvature was the sole variable that we manipulated.

All morphs were presented in random order to participants, who rated the attractiveness of each morph on a 10 -point scale $(1=\mathrm{ex}$ tremely unattractive, $10=$ extremely attractive).

\subsection{Results}

\subsubsection{Statistical analysis}

Because each participant rated the attractiveness of multiple morphs of each target, we tested the relationship between lumbar curvature and attractiveness via hierarchical linear modeling (HLM). Attractiveness ratings were clustered by participant-target combination to control for baseline differences in participants' perceptions of the targets' attractiveness. Controlling for between-target differences in attractiveness eliminated between-target influences on attractiveness (facial appearance, clothing, etc.), thereby isolating the relationship between lumbar curvature and attractiveness.

\subsubsection{Lumbar curvature and attractiveness}

To test the relationship between women's lumbar curvature and attractiveness, we fitted three different models: (1) a simple linear relationship, (2) a curvilinear relationship with an unspecified inflection point, and (3) an inverse linear relationship between women's attractiveness and their lumbar deviation from the hypothesized optimum of $45.5^{\circ}$. Model 1 differs from model 3 in that model 1 posits that increases in lumbar curvature above the hypothesized optimum are associated with further increases in attractiveness, whereas model 3 proposes that further increases above this angle are associated with decreased attractiveness as women's spines approach hyperlordosis. Although both model 2 and model 3 are consistent with an evolutionary history of stabilizing selection, model 3 more directly reflects the idea that men possess an evolved mate preference specifically for the angle 

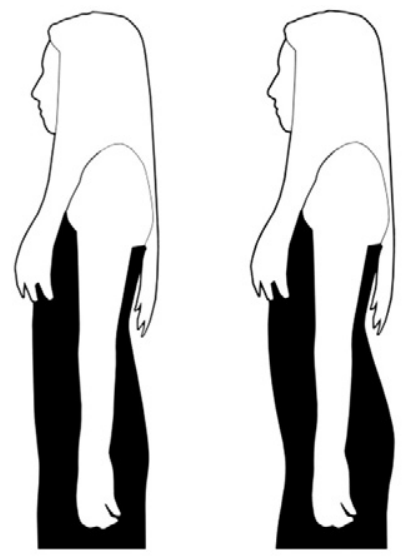

$26^{\circ}$

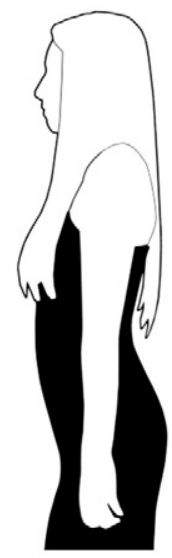

$4^{\circ}$

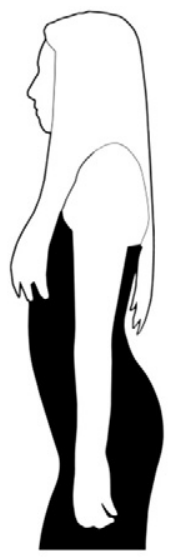

$52^{\circ}$

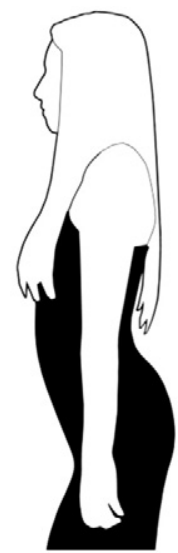

$61^{\circ}$

Fig. 2. Schematic of a complete series of stimuli.

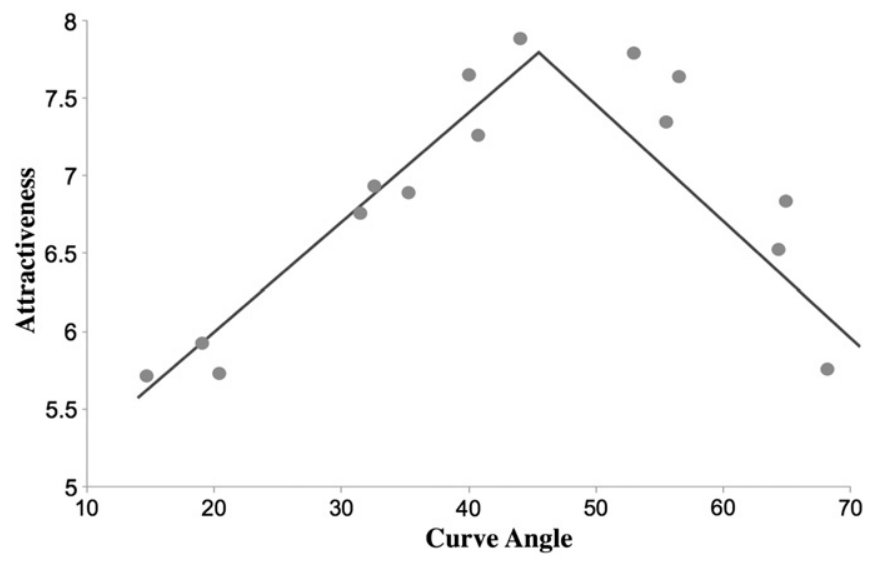

Fig. 3. The relationship between women's lumbar curvature and physical attractiveness. Dots represent mean attractiveness ratings for each morph of each model. Lines indicate model-predicted values.

of lumbar curvature maximally distant from the countervailing threats of hypolordosis and hyperlordosis. ${ }^{3}$

Among these three models, model 3 best fits the relationship between women's lumbar curvature and attractiveness (residual variances: model $1=1.80$, model $2=1.62$, model $3=1.59$ ). Precisely as hypothesized, women's attractiveness increased as their lumbar curvature approached the proposed theoretically optimal angle, $\beta=-.48, S E=.01, p<.0001$ (see Fig. 3).

\subsection{Discussion}

These data support the hypothesis that men possess an evolved mate preference for angles of lumbar curvature that facilitate the solution of the female-specific adaptive challenge of a bipedal fetal load. Precisely as hypothesized, women's attractiveness peaked at a theoretically optimal angle maximally distant from the adaptive problems of insufficient and excessive lumbar curvature.

Establishing the importance of lumbar curvature does not demonstrate, however, that this preference is specifically for cues to vertebral wedging. Both vertebral wedging and buttock mass can influence the external angle formed between the buttocks and thoracic spine-the

\footnotetext{
${ }^{3}$ Implicit in this model is the assumption that the fitness costs of hypolordosis and hyperlordosis are roughly equivalent. Although the exact probabilistic fitness costs may not be strictly identical, this model represents a reasonable operationalization of the central hypothesis.
}

clinical definition of lumbar curvature (see George et al., 2003). We therefore conducted a second study to discriminate between these two alternatives: does men's preference for lumbar curvature reflect a preference for cues to vertebral wedging, or can it be attributed to a preference for buttock mass?

\section{Study 2: a preference for cues to vertebral wedging or buttock mass?}

In Study 2, participants viewed photographic arrays depicting multiple morphs of individual female targets. Within each array, all morphs exhibited identical buttock protrusion, but this resulted from (a) vertebral wedging in one morph, and (b) buttock mass in the others.

Participants selected the most attractive morph within each array. This forced-choice procedure enabled a direct test of whether men's preference for female lumbar curvature is driven by a preference for cues to vertebral wedging or by a preference for buttock mass (e.g., Brown \& Konner, 1987).

\subsection{Method}

\subsubsection{Participants}

Two hundred two men $\left(M_{\text {age }}=18.80\right.$ years, $S D_{\text {age }}=1.50$, age range: 17-33) were recruited from the psychology subject pool at The University of Texas at Austin. Participants were granted course credit for their participation.

\subsubsection{Image arrays}

Participants were presented with 12 photographic arrays, each of which consisted of three morphs of a single female target. Within each array, all three morphs exhibited identical protrusion of the buttocks, but for different morphological reasons (see Fig. 4). The buttock protrusion of the first two morphs resulted from buttock mass. The first morph exhibited a distribution of buttock mass associated with physical fitness. The second morph exhibited a distribution of buttock mass associated with deposition of adipose tissue around the hips, a cue to fertility in women (de Ridder et al., 1990; Jasienska, Ziomkiewicz, Ellison, Lipson, \& Thune, 2004). The buttock protrusion of the third morph, however, reflected lumbar vertebral wedging.

The physical fitness and vertebral wedging morphs within a given array also exhibited an identical angle between the thoracic spine and buttocks. That is, within a given array, these morphs exhibited the same external lumbar curvature.

Controlling for these attributes among the morphs was a key feature of study design. Because all three morphs within each array exhibited 



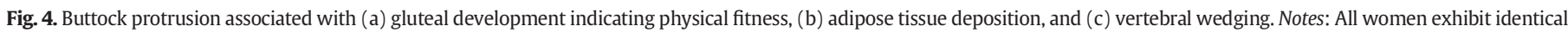
buttock protrusion. Women (a) and (c) also exhibit an identical angle between the thoracic spine and buttocks (i.e., lumbar curvature).

the same protrusion of the buttocks, any differences in attractiveness between morphs could not be attributable to buttock protrusion. Further, because the physical fitness and vertebral wedging morphs within a given array possessed the exact same angle of external lumbar curvature, any differences in attractiveness between these morphs could not be attributable to curvature angle per se, but rather must be due to the underlying morphological cause (buttock mass vs. vertebral wedging).

We generated and tested arrays at four different levels of buttock protrusion (conditions 1-4). In all arrays in all conditions, the morphs described above exhibited the exact same angle of external lumbar curvature. In some conditions, this angle was below optimum; in condition 1 , it was over $9^{\circ}$ below optimum, and in condition 2 it was approximately $4.5^{\circ}$ above optimum. Paralleling this structure, in condition 3 this angle was approximately $4.5^{\circ}$ above optimum, and in condition 4 it was approximately $9^{\circ}$ above optimum. This enabled us to test the hypothesized preference for cues to vertebral wedging at angles of external lumbar curvature both above and below the hypothesized optimum.

Our hypothesis that men have an evolved preference for cues to an optimal, intermediate degree of vertebral wedging leads to the following predictions. First, when women exhibit a degree of lumbar curvature that is below the optimum, men should prefer the woman for whom this angle results from vertebral wedging over a woman for whom this angle reflects buttock mass-in this case, the vertebral wedging morph possesses a degree of underlying wedging closer to the optimum. Second, and in contrast, when women's lumbar curvature exceeds the optimum, this preference for the vertebral wedging morph should disappear. When two women exhibit an angle of external lumbar curvature that exceeds the optimal value, the woman with greater buttock mass-for whom this angle is associated with less extreme vertebral wedging-is the one whose underlying vertebral wedging is closer to optimum. This hypothesis therefore yields the prediction that men should prefer the vertebral wedging morph at angles of lumbar curvature below the optimum, but not above the optimum.

Because the buttock mass morphs had more mass around the hips, in each array they possessed a lower WHR than the vertebral wedging morph. For each array, participants were asked to select the most attractive of the three morphs.

\subsection{Results}

We conducted chi-square analyses on the forced-choice data to determine whether the Study 1 findings reflect (1) a theoretically predicted, novel male preference for cues to vertebral wedging, or (2) the first systematic evidence for a male preference for buttock mass. Either outcome would have been empirically informative, but our a priori reasoning pointed to the importance of vertebral wedging as a theoretically anchored and previously undiscovered evolved attractiveness cue.

Overall, men preferred women whose buttock protrusion reflected vertebral wedging, not buttock mass, $\chi^{2}(2, N=202)=265.84$, $p<.0001$ (see Fig. 5). Furthermore, direct comparison of the morphs possessing identical angles between the thoracic spine and buttocks (i.e., identical lumbar curvature) revealed that men preferred the morphs for whom this angle was attributable specifically to vertebral wedging, $\chi^{2}(1, N=202)=172.22, p<.0001$. These results unambiguously demonstrate that men distinguish between women whose external lumbar curvature reflects vertebral wedging and women whose external lumbar curvature reflects buttock mass.

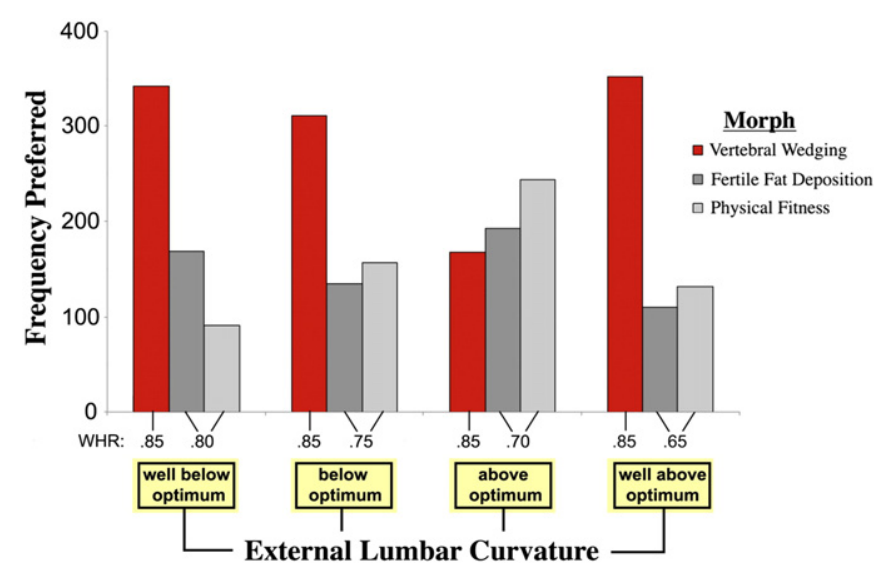

Fig. 5. Men's preference for cues to vertebral wedging versus buttock mass across different levels of external lumbar curvature. The $x$-axis organizes the arrays of women according to lumbar condition; in some conditions, the women's external lumbar curvature was below optimum, in other conditions, it was above optimum. The tick marks on the $x$-axis indicate the WHR of the women within the arrays. 
However, our theoretically anchored reasoning did not point toward a simple main effect; rather, our hypothesis suggested that men's preference for cues to vertebral wedging should depend on women's degree of external lumbar curvature. Consistent with these expectations, men's preference for vertebral wedging varied as function of the women's angle of external lumbar curvature [morph $\times$ condition interaction: $\chi^{2}(6, N=202)=182.82, p<.0001$ ] (Fig. 5). When the photographic arrays depicted women with below-optimal levels of lumbar curvature (conditions 1 and 2), men preferred women whose external lumbar curvature reflected underlying vertebral wedging over women whose (identical) external lumbar curvature reflected greater buttock mass [condition 1: $\chi^{2}(1, N=202)=144.83, p<.0001$; condition $2: \chi^{2}(1$, $N=202)=51.23, p<.0001]$. However, in condition 3 , in which the morphs exhibited above-optimal levels of external lumbar curvature, the preference for the vertebral wedging morph disappeared. At these above-optimal levels of lumbar curvature, men preferred the morph with greater buttock mass-the morph whose underlying vertebral wedging was closer to the optimum, $\chi^{2}(1, N=202)=14.02$, $p<.001$. Unexpectedly, when women exhibited angles of lumbar curvature more drastically above the optimum, the data showed a return to the pattern we observed at below-optimal levels [condition 4: $\left.\chi^{2}(1, N=202)=105.66, p<.0001\right]$. We discuss this unpredicted finding below.

\subsection{Discussion}

These results show that men's preference for lumbar curvature is unequivocally not a by-product of a preference for buttock mass. Men (1) discriminate between women whose external lumbar curvature reflects vertebral wedging and those whose (identical) curvature reflects buttock mass, (2) prefer women with greater vertebral wedging over women with greater buttock mass at sub-optimal levels of lumbar curvature-when the former's underlying spinal structures are closer to the optimum, and (3) exhibit the expected reversal of this preference at certain angles of lumbar curvature above optimum.

Along with these theoretically expected findings came an unexpected observation: men appear to prefer women with greater vertebral wedging at angles of lumbar curvature drastically above the optimum. We interpret this finding as an anti-preference, and we think at least two distinct causes may be responsible for this phenomenon. In condition 4 , the buttock mass morphs possessed less extreme vertebral wedging than the wedging morphs, but the relative benefits of this slightly reduced vertebral wedging may not offset the costs of the buttock mass morphs' excessively low WHR and extreme degree of buttock mass. That is, the buttock-mass morphs in condition 4 may have exhibited cues to the adaptive problems of both (1) excessive lumbar curvature and (2) excess lower body mass and a below-optimal WHR.

This second cluster of adaptive problems is associated with multiple fitness-related costs. Extreme accumulation of adipose tissue puts individuals at risk for obesity-related fitness costs (Friedman, 2009), and a WHR below 0.7 is associated with increased likelihood of hip fracture (Faulkner et al., 1993; Frisoli et al., 2005). In light of previous research showing that men are less attracted to women with WHRs below 0.7 (e.g., Marlowe \& Wetsman, 2001; Streeter \& McBurney, 2003), and our Study 1 finding that men are less attracted to women with angles of lumbar curvature that deviate from optimum, it may not be plausible to interpret the disappearance of men's preference for the buttock mass morphs in condition 4 as reflecting a male preference for angles of lumbar curvature well above optimal values. Rather, we suggest that men's shunning of the buttock mass morphs in condition 4 likely reflects an anti-preference for women exhibiting cues to both above-optimum lumbar curvature and a sub-optimal WHR.

For now, it appears reasonable to conclude that men's preference for lumbar curvature is not a by-product of a preference for greater buttock mass. Across all four conditions, men discriminated between women whose lumbar curvature reflected greater vertebral wedging and women whose curvature reflected greater buttock mass. In all conditions but one, men preferred the women whose lumbar curvature was closer to optimum. This included (1) preferring women exhibiting cues to greater underlying vertebral curvature in conditions in which the women exhibited below-optimal levels of curvature, and (2) preferring cues to less underlying vertebral wedging in condition 3 , when the women exhibited above-optimal levels of curvature. Moreover, the fact that men's preference for cues to greater vertebral wedging prevailed in both of the below-optimal conditions-despite the WHR advantage of the women with greater buttock mass -is a striking indicator of the robustness of this preference.

\section{General discussion}

The convergent results from these independent studies reveal a strong fit between observed standards of attractiveness and a priori predictions based on selective pressures operating during hominin evolution. These tests suggest that selection fashioned not only spinal structures in women for solving pregnancy-related challenges, but also attendant psychological adaptations in men for assessing female physical attractiveness on the basis of these structures.

\subsection{A preference for "averageness"?}

Our hypothesis about men's preference for "intermediate" lumbar curvature was driven by a priori reasoning, anchored in the adaptive problems posed to ancestral women by hypolordosis and hyperlordosis. The countervailing fitness threats of insufficient and excessive lumbar curvature would have created stabilizing selection on women's spines favoring an intermediate optimum between hypolordosis and hyperlordosis. Accordingly, we operationalized this theoretically optimal angle by reference to values identified in the orthopedic medical literature as posing these fitness-relevant threats.

Reproductive-aged women's mean angle of lumbar curvature may approximate this theoretically optimal angle of lumbar curvature, but framing men's preference merely as one for "average" values is only partially accurate ${ }^{4}$ and, more importantly, it misses the central evolutionary reason for the preference.

Humans' distant ancestors did not have the vertebral wedging present in the modern female spine; the average among our primate ancestors was distinctly non-wedged (Whitcome et al., 2007). This is because, prior to bipedalism, selection would have penalized lumbar vertebral wedging; its biomechanical costs would not have been outweighed by fetal load-offsetting benefits.

The transition to bipedalism introduced novel selective pressures that favored (a) female lumbar vertebral wedging and (b) male mate preferences for women exhibiting cues to such wedging. Precisely because of this dual (natural and sexual) selective advantage of vertebral wedging over the ancestral average, the new, wedged optimum gradually would have come to characterize the center of reproductive-aged women's distribution. Portraying men's preference as one for "average" values thus misses the central evolutionary reason underlying the preference.

Determining the nuances of the evolutionary history that led to modern humans' lumbar curvature awaits future research. At present, the results from these studies support the hypothesis that men possess theoretically anchored and previously undiscovered mate preferences for lumbar curvature, and that this reflects a preference for vertebral wedging, not buttock mass. Lumbar curvature thus represents an

\footnotetext{
${ }^{4}$ The mean of reproductively viable women-but not all women-approximates the theoretical optimum. Pre-pubertal individuals exhibit angles of lumbar curvature more than $10^{\circ}$ below optimum (Shefi, Soudack, Konen, \& Been, 2013). If men's preference were based on an average prototype constructed during ontogeny, then the reduced angles to which they are exposed in their developmental cohort should contribute to the construction of this "average" and result in a preference that deviates from (1) the theoretical optimum, (2) the mean of reproductive-aged women, and (3) that observed in the current study.
} 
important, but previously undiscovered component of physical attractiveness that fits seamlessly within a broader evolutionary logic for standards of attractiveness (Sugiyama, 2005). Collectively, these findings underscore the value of an evolutionary psychological framework for understanding human standards of attractiveness.

\section{References}

Berscheid, E., \& Walster, E. (1974). Physical attractiveness. In L. Berkowitz (Ed.), Advances in experimental social psychology (pp. 157-215). New York, NY: Academic Press.

Birkhead, T. R., \& Fletcher, F. (1995). Male phenotype and ejaculate quality in the zebra finch Taeniopygia guttata. Proceedings of the Royal Society B, 262, 329-334, http://dx. doi.org/10.1098/rspb.1995.0213.

Brown, P. J., \& Konner, M. (1987). An anthropological perspective on obesity. Annals of the New York Academy of Sciences, 499, 29-46, http://dx.doi.org/10.1111/j.1749-6632. 1987.tb36195.x.

Christie, H. J., Kumar, S., \& Warren, S. A. (1995). Postural aberrations in low back pain Archives of Physical Medicine and Rehabilitation, 75, 218-224 (Retrieved from http:// www.ncbi.nlm.nih.gov/pubmed/7717811).

de Ridder, C. M., Bruning, P. F., Zonderland, M. L., Thijssen, J. H., Bonfrer, J. M., Blankenstein, M. A., et al. (1990). Body fat mass, body fat distribution, and plasma hormones in early puberty in females. Journal of Clinical Endocrinology $\mathcal{E}$ Metabolism, 70, 888-893.

Faulkner, K. G., Cummings, S. R., Black, D., Palermo, L., Glüer, C. -C., \& Genant, H. K. (1993). Simple measurement of femoral geometry predicts hip fracture: The study of osteoporotic fractures. Journal of Bone and Mineral Research, 8(10), 1211-1217.

Fernand, R., \& Fox, D. E. (1985). Evaluation of lumbar lordosis: A prospective and retrospective study. Spine, 10, 799-803, http://dx.doi.org/10.1097/00007632-198511000-00003.

Friedman, J. M. (2009). Obesity: Causes and control of excess body fat. Nature, 459, 340-342, http://dx.doi.org/10.1038/459340a.

Frisoli, A., Jr., Paula, A. P., Pinheiro, M., Szejnfeld, V. L., Piovezan, R. D., Takata, E., et al. (2005). Hip axis length as an independent risk factor for hip fracture independently of femural bone mineral density in Caucasian elderly Brazilian women. Bone, 37, 871-875.

Gangestad, S. W., \& Scheyd, G. J. (2005). The evolution of human physical attractiveness. Annual Review of Anthropology, 34, 523-548, http://dx.doi.org/10.1146/annurev. anthro.33.070203.143733.

George, S. Z., Hicks, G. E., Nevitt, M. A., Cauley, J. A., \& Vogt, M. T. (2003). The relationship between lumbar lordosis and radiologic variables and lumbar lordosis and clinical variables in elderly, African-American women. Journal of Spinal Disorders and Techniques, 16, 200-206 (Retrieved from http://www.ncbi.nlm.nih.gov/pubmed/ 12679677).

Jasienska, G., Ziomkiewicz, A., Ellison, P. T., Lipson, S. F., \& Thune, I. (2004). Large breasts and narrow waists indicate high reproductive potential in women. Proceedings of the Royal Society B, 271, 1213-1217, http://dx.doi.org/10.1098/rspb.2004.2712.
Langlois, J. H., Roggman, L. A., Casey, R. J., Ritter, J. M., Rieser-Danner, L. A., \& Jenkins, V. Y (1987). Infant preferences for attractive faces: Rudiments of a stereotype. Developmental Psychology, 23, 363-369, http://dx.doi.org/10.1037/0012-1649.23.3. 363.

Magnora, A. (1975). Investigation of the relation between low back pain and occupation. VII. Neurologic and Orthopedic Condition. Scandinavian Journal of Rehabilitation and Medicine, 7, 146-151 (Retrieved from http://www.ncbi.nlm.nih.gov/pubmed/ 130674).

Marlowe, F. W. (2003). A critical period for provisioning by Hadza men: Implications for pair bonding. Evolution and Human Behavior, 24, 217-229, http://dx.doi.org/10.1016/ S1090-5138(03)00014-X.

Marlowe, F. W., Apicella, C., \& Reed, D. (2005). Men's preference for women's profile waist-to-hip ratio in two societies. Evolution and Human Behavior, 26, 458-468, http://dx.doi.org/10.1016/j.evolhumbehav.2005.07.005.

Marlowe, F. W., \& Wetsman, A. (2001). Preferred waist-to-hip ratio and ecology. Personality and Individual Differences, 30, 481-489.

Norris, K. (1993). Heritable variation in a plumage indicator of viability in male great tits Parus major. Nature, 362, 537-539, http://dx.doi.org/10.1038/362537a0.

Petrie, M. (1994). Improved growth and survival of offspring of peacocks with more elaborate trains. Nature, 371, 598-599, http://dx.doi.org/10.1038/371598a0.

Rath, J. D., \& Rath, W. (1997). Mechanical low back pain in pregnancy-Preliminary findings in a consecutive cases series investigation. Philadelphia, PA: Fifth Internationa McKenzie Conference (Retrieved from http://duffyrath.com/pdf/Mechanical\%20Low\% 20Back\%20Pain\%20in\%20Pregnancy.pdf).

Shefi, S., Soudack, M., Konen, E., \& Been, E. (2013). Development of the lumbar lordotic curvature in children from age 2 to 20 years. Spine, 38(10), 602-608.

Singh, D., \& Singh, D. (2011). Shape and significance of feminine beauty: An evolutionary perspective. Sex Roles, 64, 723-731, http://dx.doi.org/10.1007/s11199-011-9938-z.

Stacey, L. W., Eileen, A. K., Rebecca, J. S., \& Kevin, J. M. (2011). Pterin-based ornamenta coloration predicts yolk antioxidant level in female striped plateau lizards (Sceloporus virgatus). Journal of Animal Ecology, 80, 519-527, http://dx.doi.org/10. 1111/j.1365-2656.2010.01801.x.

Streeter, S. A., \& McBurney, D. H. (2003). Waist-hip ratio and attractiveness: New evidence and a critique of "a critical test". Evolution and Human Behavior, 24, 88-98.

Sugiyama, L. S. (2005). Physical attractiveness in adaptationist perspective. In D. M. Buss (Ed.), The handbook of evolutionary psychology (pp. 292-343). New York, NY: Wiley.

Symons, D. (1995). Beauty is in the adaptations of the beholder: The evolutionary psychology of human female sexual attractiveness. In P. R. Abramson, \& S. D. Pinkerton (Eds.) Sexual nature, sexual culture (pp. 80-118). Chicago, IL: University of Chicago Press.

Thornhill, R., \& Gangestad, S. W. (1999). Facial attractiveness. Trends in Cognitive Science, 3, 452-460.

Whitcome, K. K., Shapiro, L. J., \& Lieberman, D. E. (2007). Fetal load and the evolution of lumbar lordosis in bipedal hominins. Nature, 450, 1075-1078, http://dx.doi.org/10. 1038/nature06342.

White, A. A., \& Punjabi, M. M. (1990). Clinical Biomechanics of the Spine. Philadelphia, PA: Lippincott. 